\title{
REFLEXIONES SOBRE CONFLICTOS SOCIOAMBIENTALES EN LA PRÁCTICA PEDAGÓGICA DE LA LICENCIATURA EN BIOLOGÍA:UN ELEMENTO EMERGENTE PARA ARTICULAR A LA PEDAGOGÍA PARA LAPAZ
}

\section{Reflections Towards Socioenvironmental Conflict in the Pedagogical Practice from Biology Degree Teachers: an Emerging Element to Articulate to the Pedagogy of Peace}

\section{Reflexões sobre conflitos socioambientais no estágio da Licenciatura em Biologia: elemento emergente de articulação com a pedagogia para A Paz}

Adriana E. Tovar-Martínez ${ }^{1}$

María Angélica Molina Albarracín²

Héctor Guzmán Suárez ${ }^{3}$

Fecha de recepción: $1 .^{\circ}$ de agosto 2017

Fecha de aceptación: 25 de mayo de 2018

El grupo de estudio Pedagogía sobre los Conflictos Socioambientales Colombianos, de la Licenciatura en Biología de la Universidad Pedagógica Nacional (UPN) ha sido un espacio de encuentro para reflexionar acerca de la importancia de que los maestros en formación reconozcan los conflictos socioambientales existentes en los sitios donde vienen desarrollando su práctica pedagógica y didáctica o sus trabajos de grado. La identificación de las tensiones entre actores sociales presentes en los territorios, sus intereses por la apropiación, la significación y la distribución de las riquezas provenientes de la naturaleza es un análisis necesario dentro del ejercicio de contextualización que realizan los maestros investigadores.

1 Magíster en Conservación de Vida Silvestre. Docente del Departamento de Biología de la Universidad Pedagógica Nacional. Correo electrónico: aetovarm@pedagogica.edu.co

2 Licenciada en Biología. Magister en Tecnologías de la Información Aplicadas a la Educación. Docente del Departamento de Biología de la Universidad Pedagógica Nacional. Correo electrónico: mamolina@pedagogica.edu.co.

3 Antropólogo de la Universidad Nacional de Colombia. Docente del Departamento de Biología de la Universidad Pedagógica Nacional. Correo electrónico: hlguzmans@pedagogica.edu.co. 
Los trabajos de práctica pedagógica y de grado que se han desarrollado desde las dos líneas de investigación del grupo ascienden a más de 40 . En ellos se han abordado contextos y comunidades diversas del territorio colombiano, entre los que se encuentran las comunidades indígenas de la baja bota caucana y del municipio de Sotará, en el departamento del Cauca; del municipio de Gurayaco en el Caquetá; en los municipios de Puerto Nariño, La Chorrera y Leticia, en el departamento del Amazonas. Comunidades negras del municipio de Bahía Solano en el Chocó, Puerto Asís en Putumayo, y Bocas de Satinga, en Nariño; y comunidades campesinas del Valle de Tenza en Boyacá, y de diversos municipios de Cundinamarca.

Aquí el reconocimiento del contexto educativo social, cultural y político ha sido un común denominador en todos los trabajos investigativos desarrollados en la línea. Se entiende entonces que el reconocimiento del contexto permiten identificar horizontes de sentido que desafían el actuar del docente (Universidad Pedagógica Nacional, UPN, 2014); y se considera que las acciones que, como seres humanos, realizamos sobre la naturaleza tienen implicaciones éticas, económicas y políticas (Castaño, 2011). Esto ha posibilitado que los maestros se posicionen como investigadores que comprenden las realidades locales, reconocen el territorio y propician su apropiación. Es aquí en donde los conflictos socioambientales han emergido, tal vez con otros nombres, como elementos que forman parte de la memoria biocultural de las comunidades, configurando su plan de vida y de abundancia. Situaciones como la explotación insostenible de los manglares de Bocas de Satinga (Angulo y Arias, 2017) y en Bahía Solano (Rodríguez, 2015), el reemplazo de prácticas tradicionales por cultivos ilícitos en la baja bota caucana, y la desacreditación de los saberes campesinos cundinamarqueses dada la presión urbana por el desarrollo económico (Muñoz, 2017) son temas pertinentes que se deben abordar desde la reflexión pedagógica contextualizada.

La discusión sobre los conflictos socioambientales en el marco del posacuerdo toma relevancia cuando partimos de reconocer la relación indisoluble entre conflicto armado y naturaleza. En la historia colombiana se evidencian estos vínculos complejos e incluso contradictorios, pues obedecen a los intereses y acciones de los actores del conflicto. Rodríguez G., Rodríguez F. y Durán (2017) del grupo Dejusticia ${ }^{4}$ reconocen cuatro tipos de vínculos entre los recur-

4 El grupo Dejusticia constituye un centro de estudios jurídicos y sociales, de Bogotá, cuyo objetivo es la realización de estudios rigurosos, el desarrollo de propuestas políticas y acciones jurídicas a favor del fortalecimiento del Estado de derecho y la promoción de los derechos humanos en Colombia. sos naturales y el conflicto armado: los recursos naturales causa de origen del conflicto armado, una de sus fuentes de financiamiento y perpetuación, una víctima, y paradójicamente un beneficiario de este. De esta manera, proyectar la construcción de una paz estable y duradera requiere asumir la reflexión sobre los conflictos socioambientales y alternativas para su abordaje en contextos escolares. Cabe aclarar que la intención de esta reflexión no es discutir la coherencia en lo pactado en el acuerdo final, ni tampoco entrar en detalles de las dificultades de su implementación. El propósito es identificar los aportes de la educación y reflexión pedagógica a las relaciones que configuran la praxis educativa en la construcción de una sociedad para la paz en el marco del posacuerdo.

El 27 de marzo de 2017 en el Departamento de Biología de la UPN, sede Bogotá, en el transcurso de un Seminario de Práctica Pedagógica y durante un taller pautado por la Línea de investigación Pensamiento Crítico, Política y Currículo, una estudiante, sintetizando el espíritu del debate que se llevaba a cabo en ese momento (y haciendo eco de las discusiones generadas a partir del análisis de las prácticas educativas en distintos contextos educativos del país) manifestaba que la biología, que tradicionalmente se ha "movido" desde el lugar del saber de lo vivo, desde hace ya algún tiempo para acá se ha estado "moviendo" hacia lugares que la trascienden como disciplina. Expresaba que, en el caso concreto de temas como el de la conservación, mientras algunos consideran que debe seguirse planteando desde unas ideas de biodiversidad y naturaleza ya establecidas, otros proponen que se debe concebir más desde el cuidado de la vida, que eso no se puede hacer desconociendo los saberes de las comunidades, y que todo ello implica hacer referencia a una serie de valores que aún no se han "mapeado".

En este sentido, el grupo de investigación viene reflexionando sobre qué es lo que hace que un Licenciado en Biología sea un maestro, reconociendo por un lado que el saber disciplinar biológico implica la capacidad o habilidad de potenciar unas relaciones con el otro, y, por otro, que el saber disciplinar pedagógico se constituye no desde el poder del que sabe sino desde el diálogo de saberes, tejiendo las experiencias en clave de hospitalidad. Así, un maestro "se preocupa por construir relaciones de alteridad", y si los profesores somos sujetos de podery saber es porque "podemos construir con el otro".

La educación, la enseñanza, es experiencia, es pasión que solicita otro lenguaje. Un lenguaje capaz de incorporar la incertidumbre, lo singular, los saberes y sus sombras, las decepciones, imposibilidades y alegrías. La propia subjetividad expuesta a la contingencia de su 
trabajo, reconstruyendo simultáneamente, el paisaje exterior de la acción y ese paisaje interior de pensamientos e intenciones. (Rattero, 2011, p. 184).

Como bien se señalaba en el evento mencionado, la posibilidad del maestro de construir y propiciar relaciones de alteridad con el otro es un aporte a la construcción de paz. El reconocimiento del otro, de aquello que tenemos en común y de aquello en lo que diferimos, nos permite acercarnos a la convivencia en la diferencia de identidades. Pero, ¿qué tiene que ver esto con el conflicto socioambiental y la construcción de una paz estable y duradera? Proponemos que, en nuestra sociedad, dicha alteridad se extienda intencionalmente a los ecosistemas y a otros seres vivos no humanos. Esta posibilidad ya había sido evidenciada por autores como Arhem (1993), y le permite concluir que "Para el mundo industrializado y secularizado, tal vez haya aquí una lección por aprender: el que el conocimiento ecológico debe ser investido de valor moral y poder emocional para motivar la acción social y guiar el comportamiento económico [...]” (p. 126).

Los maestros pueden generar procesos educativos para el reconocimiento de lo vivo, de la interdependencia, de la reciprocidad y de la vida como experiencia del mundo. La transformación de la cultura hacia la paz será posible en la medida en que podamos construir nuevas formas de relacionarnos con lo vivo, de propiciar otras maneras de pensar el mundo y experienciarlo a través de relaciones de respeto. El maestro como sujeto social y político se configura como constructor de una nueva ética que permita responsabilizarnos de nosotros mismos y de nuestras interacciones con lo vivo. Al respecto, el filósofo Guillermo Hoyos propone construir una ética dialógica, en la que la comunicabilidad se extienda a "los relatos emergentes de un contexto, de una región, de un grupo social minoritario, de una especie en extinción, de un paisaje, de voces menores" (citado en Noguera, 2007, p. 2). Por ello, es vital explorar cómo se han constituido culturas alrededor de modos de entender las formas de apropiación de la naturaleza por parte de diversos actores presentes en los territorios donde el conflicto armado ha tenido cabida. Asimismo, es importante reconocer aquellas epistemologías en las cuales los grupos humanos han generado un vínculo vital con la naturaleza infraccionable y no dualista, ya que ello nos permitirá reconocer otras formas de establecer nuestras prácticas y nuestras formas de estar en el mundo.

Nuestro lugar como maestros es el de promover el diálogo de conocimientos entre las disciplinas que nos corresponden y los conocimientos que han construido las comunidades locales del territorio colombiano (Santos, 2012). Este diálogo, como sugiere Freire (2007), debe sustentarse desde realidades concretas, de orden social, económico y político. Esta presencia es primordial en áreas de alta biodiversidad donde surgirán nuevos conflictos por el uso de la naturaleza, por ejemplo las áreas catalogadas como reserva forestal que se otorgarán como parte del fondo para la sustitución de tierras, o los territorios de comunidades indígenas y afro en donde el sector minero-energético puede tener intereses de producción.

Para poder aportar desde los diversos escenarios educativos a la construcción de una paz incluyente, interdisciplinar, intercultural e incluso intergeneracional, proponemos resignificar nuestras concepciones diversas de la naturaleza, del otro no humano, para que sea posible que aquellas características principales y propósitos que se formulan sobre la pedagogía para la paz se puedan extender a la naturaleza, de manera que formas de vida no humana también tengan un lugar de respeto, reparación y reconocimiento. Igualmente, planteamos que se promueva un diálogo de conocimientos que propenda por el fortalecimiento de las comunidades, las prácticas que favorecen la resiliencia y adaptación a las dinámicas ecológicas de los ecosistemas y el cambio de las prácticas que atenten inoficiosamente contra la biodiversidad; como lo denomina el profesor Gustavo Wilches, hacer gestión al "paz-conflicto" (Wilches-Chaux, 2016).

Desde una perspectiva intercultural, una primera propuesta de reflexión pedagógica en torno al conflicto socioambiental es la de reconocer que lo que ha hecho posible la supervivencia de las culturas ancestrales es el reconocimiento del territorio, el desarrollo de la capacidad de coexistencia con él de una forma adaptativa. El relato de las comunidades anfibias de la depresión momposina presentado por Fals Borda en Historia doble de la costa (Fals-Borda, 1984), es una muestra de ello. Las cosmovisiones indígenas rompen con la idea de naturaleza como recurso y permite repensar la posición que tiene el ser humano en la vida y desde su relación con el entorno, legitimando otras formas de interacción con el otro no-humano.

Así por ejemplo, los desana en su "pensar-día" se consideran cuidadores, guardianes o protectores de un territorio que les ha sido dado, confiado, otorgado, y que deben mantener equilibrado, puro y sano para asegurar la continuidad de la vida y que las futuras generaciones lo puedan a su vez disfrutar y legar. Los principios son la interdependencia y la reciprocidad, porque no solo perciben la dimensión física del paisaje con sus ojos, sino también su dimensión energética y su dimensión cronológica o temporal de manera multisensorial, sus propiedades y efectos (Reichel-Dolmatoff, 1993). 
De manera similar, para los makuna en la vida cotidiana los principios de interdependencia y reciprocidad son determinantes. Esto tiene relación con la manera en la que perciben a los seres vivos; estos conforman tres grandes grupos: yai o "aquellos que se alimentan de nosotros", masa o "la gente", y wai o "nuestra comida", pero la pertenencia a cada uno no es algo fijo, invariable, sino que es una cuestión de contexto; ser el comedor, ser "la gente", o ser la comida es solo un asunto de circunstancias (Arhem, 1993).

La importancia del análisis de los conflictos socioambientales en la construcción de tejido social teniendo como propósito el fin a la guerra en Colombia parte de entender el lugar de la educación para comprender la vida y los retos que implica su cuidado, lo cual exige explorar en la afectividad, reconstruir nuestra forma de entender el cuerpo y su dimensión colectiva.

Es así como la enseñanza de la biología para Colombia trasciende la perspectiva de los contenidos, y con ello, es una labor que no puede continuar haciéndola personas que asumen su condición de maestros solo por el dominio de los contenidos disciplinares. Debe ser un sujeto con gusto por lo humano, con un alto deseo de encuentro con el otro, un sujeto que piensa su acción en clave de la cultura, de la vida, de la política, de apertura a la diversidad, del reconocimiento de la vida como auto-afección, del reconocimiento de sí en una relación integral, en la cual se reconozca el ser con el otro, y desde allí se defina y oriente la práctica pedagógica y dinamice el currículo. Consciente también de las realidades laborales de los maestros y desde sus prácticas cotidianas de vida.

Por otro lado, es necesario considerar la importancia de que los maestros conozcan el acuerdo final, se conciban como sujetos políticos capaces de participar en escenarios educativos y procesos comunitarios, así como pensar los puntos del acuerdo desde una mirada educativa y ambiental. El debate sobre los mecanismos, posibilidades y limitaciones a la hora de la participación de los maestros de biología es amplio y se está configurando; sin embargo, consideramos pertinente iniciar el debate frente a uno de los elementos más alarmantes en materia ambiental en el marco del posacuerdo: el otorgamiento de tierras y la implementación de procesos productivos con aquellas comunidades beneficiarias. En el primer punto se habla de democratización del acceso y uso adecuado de la tierra, en donde el Fondo de Tierras dispondrá de tres millones de hectáreas, entre las que se incluyen reservas forestales. Su uso está condicionado a la formulación de "planes que garanticen sostenibilidad social y ambiental" (Wilches-Chaux, 2016, p. 3). En función con el artículo 58 de la Constitución política, dichas áreas deben cumplir una función ecológica que traduce, según Macías, en la generación de riquezas y desarrollo "dentro de un marco de protección ambiental, estableciendo limitaciones o condicionamientos al derecho de uso pero no impidiendo este derecho" (2014, p. 133). En caso de que el Gobierno no cumpla con una correcta implementación del acuerdo, garantizando condiciones de vida dignas para las comunidades, los bienes y servicios ecosistémicos (BSE) estarían en riesgo y por tanto ocasionarían el detrimento de un interés colectivo, así como afectaciones directas a la fauna silvestre y los ecosistemas vulnerables y estratégicos de nuestro país. Es claro que un maestro capaz de favorecer el reconocimiento y la apropiación del territorio a través de prácticas tradicionales adaptadas a las dinámicas de los ecosistemas puede marcar una diferencia.

Teniendo en cuenta que la UPN tiene el compromiso de investigar el papel de la educación en una sociedad que sepa tramitar sus conflictos por la vía del diálogo, consideramos que se aporta a la construcción de una paz estable y duradera desde el reconocimiento del docente como actor estratégico en el abordaje y la resolución de los conflictos socioambientales.

Proponemos abordar el reconocimiento de los conflictos socioambientales para posibilitar la reflexión frente a lo vivo y la vida, como elemento articulador de la pedagogía para la paz. Finalmente, es importante señalar que hay mucho por discutir, por ejemplo ¿Cómo abordar los conflictos socioambientales? ¿Cómo articularlos con la enseñanza de la biología? ¿De qué forma su abordaje aporta al fortalecimiento de las territorialidades en las comunidades?

\section{Referencias}

Angulo Q., K. y Arias, M. (2017). Cultivando pensamiento para la vida entre las raíces de Bocas de Satinga, una experiencia de práctica integral de pesca y manglares (trabajo de grado). Universidad Pedagógica Nacional, Bogotá, Colombia.

Arhem, Kaj. (1993). Ecosofía makuna. En F. Correa (ed.). La selva humanizada. Ecología alternativa en el trópico húmedo colombiano. Bogotá: Instituto Colombiano de Antropología (ICAN), Fondo FEN Colombia, Fondo Editorial Cerec.

Castaño, N. C. (2011). Enseñanza de la biología y diversidad cultural. Bio-grafía 6(11):7-10.

Fals-Borda, O. (1984). Historia doble de la costa (vol. 1). Bogotá: Carlos Valencia.

Freire, P. (2007). Educación como práctica de la libertad. México: Tierra Nueva. 
Macías G., L.F. 2014. Función social y función ecológica de la propiedad, En A. Sáenz Q; A. Gómez R.; G. A. Rodríguez (Eds.). Conflictos entre propiedad, comercio y ambiente. Grúpo Editorial Ibañez. Bogotá.

Muñoz, M. 2017. Orientaciones pedagógicas y didácticas en la ruralidad: un posible camino del maestro de biología en el campo (trabajo de pregrado). Universidad Pedagógica Nacional, Bogotá, Colombia.

Noguera, P. (2007). Horizontes de la ética ambiental en Colombia: de las éticas ambientales antropocentristas a las éticas ambientales complejas. ISEE, PUblicación Ocasional (1):1-5. Recuperado de http:// www.cep.unt.edu/papers/noguera-sp.pdf.

Palacio, G. (2001). Introducción. En: G. A. Palacio Castañeda (ed.). Naturaleza en disputa, ensayos de historia ambiental de Colombia 1850-1995. Bogotá: Facultad de Derecho, Ciencias Políticas y Sociales, Universidad Nacional de Colombia; Instituto Colombiano de Antropología e Historia, Icanh.

Pérez-Rincón, M. (2015). Conflictos ambientales en Colombia: actores generadores y mecanismos de resistencia comunitaria. Ecología política (pp. 7682). Recuperado de http://www.ecologiapolitica. info/? $p=1980$.

Rattero, C. (2011). La pedagogía por inventar. En: C. Skliar y J. Larrosa (comp.). Experiencia y alteridad en educación. Rosario: Homo Sapiens.

Reichel-Dolmatoff, G. (1993). Algunos conceptos de los indios desana del Vaupés sobre manejo ecológico. En: F. Correa (ed.). La selva humanizada. Ecología alternativa en el trópico húmedo colombiano. Bogotá: ICAN, Fondo FEN Colombia, Fondo Editorial Cerec.
Rodríguez, G. F. (2015). Orientaciones pedagógicas para la apropiación del territorio en estudiantes de los grados tercero a quinto de la Institución Educativa Luis López Mesa a partir del reconocimiento del ecosistema de manglar de Ciudad Mutis (Bahía Solano), Chocó (trabajo de pregrado). Universidad Pedagógica Nacional, Bogotá, Colombia.

Rodríguez G., C.; D. Rodríguez F.; H. Durán Crane. (2017). La paz ambiental, retos y propuestas para el posacuerdo. Documentos Dejusticia 30. Bogotá.

Santos, B. de S. (2012). De las dualidades a las ecologías. Red Boliviana de Mujeres Transformando la Economía, Remte. La Paz, Bolivia: Punto de Encuentro.

Universidad Pedagógica Nacional. (2014). Plan de Desarrollo Institucional 2014-2019: Una universidad comprometida con la formación de maestros para una Colombia en paz. Bogotá: autor.

Wilches-Chaux, G. (2016). Base ambiental para la paz. La necesidad de hacerle gestión del riesgo al paz-conflicto. Estudio técnico elaborado para el proyecto Agenda Común para la Paz. Bogotá: Planeta Paz-Oxfam. 
